\title{
Los costos de la violencia en El Salvador ${ }^{1}$
}

\author{
Luis Ernesto Romano $\sigma^{2}$
}

\section{Resumen}

El presente artículo expone de manera breve los resultados de un cálculo sobre lo que le cuesta al país anualmente el problema de la violencia. Este cálculo se ha realizado siguiendo una metodología de medición de costos propuesta por el BID y la OPS. El ejercicio, que incluye tanto los costos directos —en salud y seguridad-, como los indirectos -en pérdidas a la economía doméstica y nacional-arroja que en 1995, los costos de la violencia en El Salvador ascendieron a casi 800 millones de dólares, lo que significa cerca del 13 por ciento del Producto Interno Bruto de ese año.

En esta sinopsis se presentan los costos de la violencia que han sido medidos a través de la metodología discutida para tal efecto a iniciativas del Banco Interamericano de Desarrollo (ver Bobadilla et al, 1995). Por lo mismo, para estimar lo que cuesta la violencia a la sociedad salvadoreña se considerarán los costos directos e indirectos de la violencia para, de esa manera, abarcar no solamente aquellas cifras asociadas a la atención de la violencia en sí misma, sino también los costos sociales en que se incurre debido a los cambios producidos por el evento violento. En los párrafos siguientes se presentan algunas estimaciones y cifras oficiales en torno a los costos de la violencia, obtenidos principalmente a partir de las Encuestas de Hogares de Propósitos Múltiples (EHPM) del Ministerio de Relaciones Exterio-

1. Este artículo constituye un resumen del segundo capf́tulo del informe de investigación "La violencia en El Salvador en los noventa", el cual ha sido realizado bajo los auspicios del Banco Interamericano de Desarrollo (BID) dentro del Proyecto de Red Regional de Centros de Investigación.

2. Analista económico del Centro de Información, Documentación y Apoyo a la Investigación (CIDAI) de la UCA. 
res, Promoción y Cooperación Internacional, en el caso de los costos personales, y del Presupuesto General de la Nación, el cual ofrece valiosa información sobre costos institucionales y de rehabilitación.

\section{Costos directos}

Los costos directos se han dividido según la propuesta de índice formulada por el Banco Interamericano de Desarrollo, y en el cual se clasifican los costos con base en el sector impactado, así se subdividieron los costos directos en ocasionados al sector salud y ocasionados al sector seguridad. En ambos casos se consideran por separado los costos personales y los institucionales, con la finalidad de distinguir entre los costos incurridos por agentes privados y por el Estado.

\subsection{Sector salud}

\section{A. Costos personales o en el hogar}

Existen diferentes costos que son considerados en esta sección: consulta, transporte exámenes y medicinas. Los principales insumos para esta sección han sido obtenidos a partir de las Encuestas de Hogares Múltiples de Propósitos Múltiples para el año 1996. Los resultados de la EHPM (Dirección de Información, 1997) muestran un número extremadamente bajo de personas lesionadas para 1996: 1,230 personas; sin embargo, la misma encuesta revela que en cada caso, la lesión significó un costo promedio de 343 dólares, entre el gasto de consulta (atención ambulatoria y/o hospitalización), transporte, exámenes médicos y medicinas.

Cabe señalar, sin embargo, que existe un importante cuestionamiento para las cifras anteriores sobre todo en el rubro de la magnitud de los lesionados, independientemente de los supuestos y la metodología que se hayan asumido para obtenerlas. El principal cuestionamiento estriba en los datos de magnitud de la violencia encontrados en la primera parte de esta investigación, los cuales consignan una cifra de lesionados muy superior a las cifras consignadas en la EHPM. No se puede asumir, desde ningún punto de vista, que el total de lesionados no sea mayor de 2,000 casos, ni siquiera para aquellos lesionados graves que utilizaron la mayor parte de recursos del sistema? ${ }^{3}$. Una forma de subsanar este subregistro es considerar las cifras de magnitud obtenidas de las estimaciones realizadas y aplicarles el promedio de gastos reportados por la EHPM para obtener una cifra aproximada de lesionados que sí incurrieron en gastos personales. Estos resultados se muestran en el Cuadro 1 y a partir de ellos se ofrece un estimado del total de gastos personales. Si la cifra estimada de lesionados para el año pasado asciende a 78,726 personas con lesiones leves o graves por violencia intencional y el promedio de gastos personales según la EHPM es de 343 dólares, en total, los costos personales de salud de los lesionados por la violencia estarían alrededor de los 27 millones de dólares.

\section{Cuadro 1}

Estimación de los costos personales por lesiones intencionales

\begin{tabular}{ccc}
$\begin{array}{c}\text { Número de lesionados al año } \\
\text { según estimación }\end{array}$ & $\begin{array}{c}\text { Promedio de gasto (consulta, } \\
\text { transporte, exámenes y medicinas }\end{array}$ & $\begin{array}{c}\text { Costo total anual } \\
\text { anual }\end{array}$ \\
\hline 78,726 & 343 dólares & $27,003,018$ dólares \\
\hline
\end{tabular}

Fuente: elaboración propia.

3. De hecho, la EHPM sólo registra aquellos casos en que la gente consideró necesaria atención médica; se sabe que mucha población es víctima de algún delito en la cual resulta lesionada pero por diversas razones no acude al sistema. 


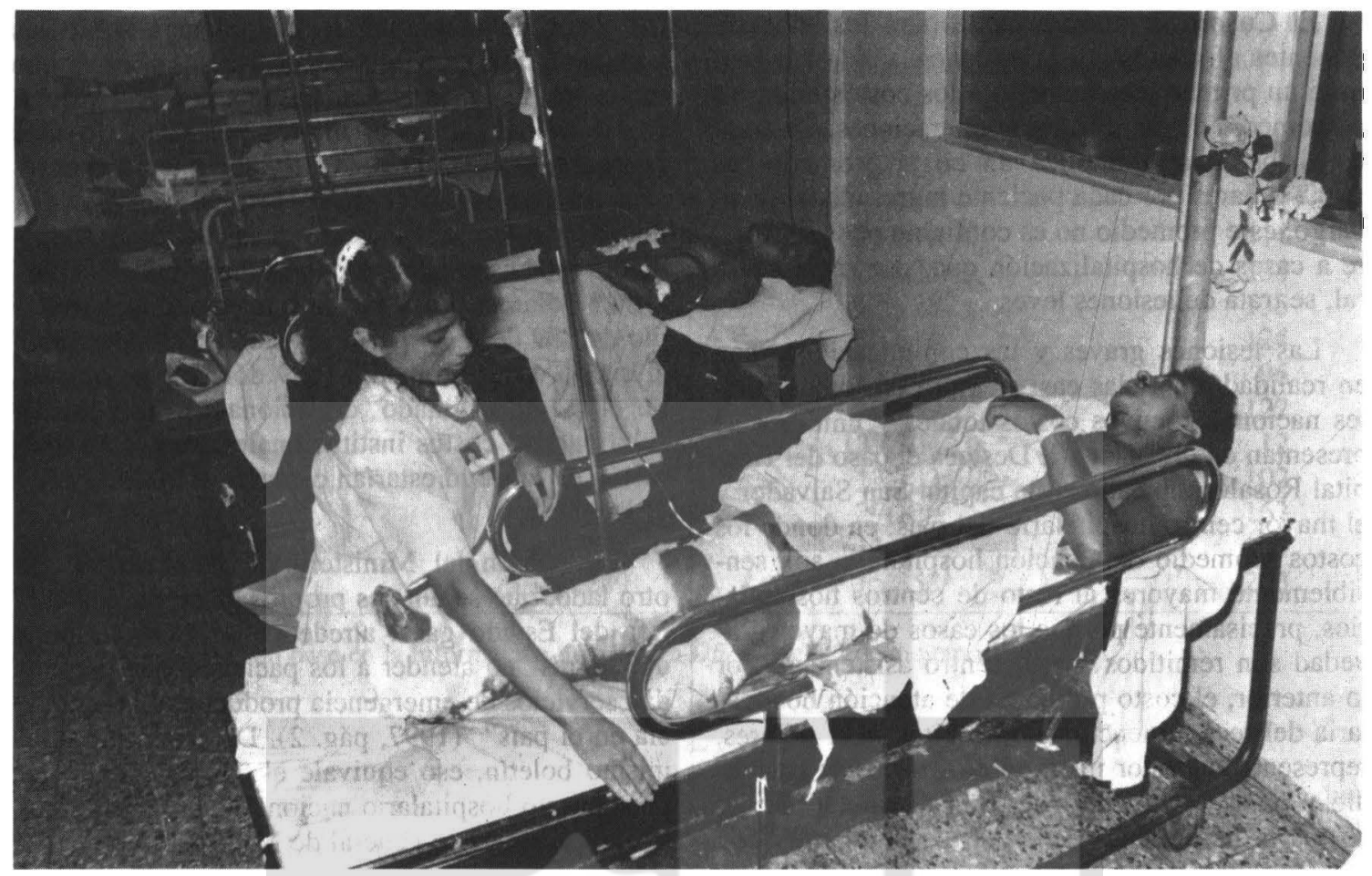

\section{B. Costos institucionales}

Para el cálculo de estos costos se ha optado por evaluarlos con base en dos métodos diferentes propuestos previamente en otros estudios (Bobadilla et al., 1995): el método de la contabilidad histórica y el método de los costos estándar. La primera persigue establecer el costo promedio de la atención de las víctimas de la violencia reflejados en el Presupuesto General de la Nación para 1996, para luego aplicarlos a los datos de magnitud de lesionados presentados en el primer capítulo de este estudio. Los costos estándar difieren de los recién descritos en que el costo promedio se calcula con base en promedios internacionales usualmente aceptados y que aíslan cualquier diferencia que podría provenir de la mayor o menor eficiencia en el uso de los recursos para la atención de la salud (Bobadilla et al., 1995).

\section{Costos contables}

El Cuadro 2 muestra la proporción del presupuesto total del Ministerio de Salud Pública y Asistencia Social (MSPAS) que es cmpleado en cada unidad presupuestaria, lo cual resulta de utilidad para filtrar los costos institucionales de salud atribuibles directamente a la violencia. Es precisamente el rubro "Recuperación de la Salud" el que contiene los gastos atribuibles a los efectos de la violencia.

\section{Cuadro 2}

Ramo de salud: destino del gasto por unidad presupuestaria (En dólares)

Unidad presupuestaria Fondo general Porcentaje

Dirección y administración institucional Atención preventiva de la salud $9,923,828$ 6.6

Recuperación de la salud $33,007,878$

Salud ambiental $19,185,614$

Apoyo a instituciones adscritas $7,840,703$

Desarrollo de la infraestructura $76,535,987$ 50.8

\section{Total}

$4,113,714$

Fuente: elaborado con base en información del Diario Oficial del 22 de diciembre de 1995, p. 218. 
El Cuadro 3 muestra cuáles son los subcomponentes afectados por la violencia y a la vez permite un primer acercamiento a los costos unitarios o promedio de las diferentes operaciones. La atención hospitalaria implica un costo promedio de 106.8 dólares por cada paciente ingresado, sin embargo, este promedio no es confiable pues se refiere a casos de hospitalización que, por regla general, se trata de lesiones leves.

Las lesiones graves y las complicaciones son en realidad atendidas casi siempre en los hospitales nacionales, cuyos costos totales y unitarios se presentan en el Cuadro 4. Destaca el caso del Hospital Rosales, ubicado en la capital San Salvador y el mayor centro hospitalario del país, en donde los costos promedio de atención hospitalaria son sensiblemente mayores al resto de centros hospitalarios, precisamente porque los casos de mayor gravedad son remitidos a este centro asistencial. Por lo anterior, el costo promedio de atención hospitalaria del recién mencionado hospital, 411 dólares, representa el valor máximo en los costos promedio y podría servir de base para el cálculo de los costos institucionales de salud al conjugarlos con los datos de magnitud de la violencia sobre las personas ${ }^{4}$. Sin embargo, hacer eso significaría que todos los lesionados han pasado por el Hospital Rosales, lo cual no es cierto; por tanto, lo más razonable sería promediar el valor máximo representado por los costos en el Hospital Rosales (411 dólares) y el valor mínimo representado en el promedio total asignado a todos los hospitales del país (106.8 dólares). Esto da como resultado un promedio de 259 dólares por costo unitario. Al asumir el citado costo promedio ( 259 dólares) y considerar el monto estimado de lesionados $(78,726)$ resulta que los costos institucionales para la recuperación de la salud estarían cercanos a los 20, 382 y 161 dólares.

Un boletín del Ministerio de Salud revela, por otro lado, que según sus propios cálculos, esa cartera del Estado gasta alrededor de 18.9 millones de dólares "en atender a los pacientes que demandan servicios de emergencia producto de la violencia en el país" (1997, pág. 2). De acuerdo cort el mismo boletín, eso equivale al 21 por ciento del presupuesto hospitalario nacional y al 12 por ciento del presupuesto general de MSPAS.

\section{Cuadro 3}

Unidad presupuestaria recuperación de la salud relación de propósitos con los recursos (En dólares)

\begin{tabular}{|c|c|c|c|c|}
\hline Línea de trabajo & Propósito & Meta* & Costo & Costo unitario \\
\hline Atención ambulatoria & Consulta médica & $1,884,991$ & $11,165,500$ & 5.92 \\
\hline Atención hospitalaria & $\begin{array}{l}\text { Servicios de } \\
\text { hospitalización }\end{array}$ & $\begin{array}{r}72,318 \\
1,000\end{array}$ & $\begin{array}{r}7,728,085 \\
206,315\end{array}$ & $\begin{array}{l}106.86 \\
206.32\end{array}$ \\
\hline $\begin{array}{l}\text { Programa de } \\
\text { reinserción } \\
\text { de lisiados de guerra }\end{array}$ & $\begin{array}{l}\text { Rehabilitación y } \\
\text { capacitación } \\
\text { Centros rehabilitación }\end{array}$ & 3 & 85,714 & $28,571.33$ \\
\hline Total & & & $19,185,614$ & \\
\hline
\end{tabular}

* A excepción del caso de los Centros de Rehabilitación cuya unidad de medida es el número de edificios, las metas de los demás rubros están expresadas en números de personas atendidas.

Nota: para la ciudad de México en 1995, los siguientes valores promedio fueron asumidos: costo promedio por individuo, 1,971 dólares; lesiones leves, desde 28 a 400 dólares; lesiones graves, desde 400 a 8,000 dólares; complicaciones, más de 25,000 dólares.

4. Este promedio se acerca al límite superior de las lesiones consideradas como leves para el caso mexicano (ver Fundación Mexicana de la Salud, 1997). 


\section{Cuadro 4}

Hospitales nacionales, costos totales y promedios de los servicios hospitalarios

\begin{tabular}{lrrr}
\hline Hospital & Costo total & Meta & Costo promedio \\
\hline Rosales & $10,549,242$ & 25,670 & 411 \\
Santa Ana & $5,922,107$ & 25,435 & 233 \\
Ahuachapán & $1,878,760$ & 13,928 & 135 \\
Sonsonate & $2,302,701$ & 18,614 & 124 \\
Chalatenango & 6999,080 & 7,300 & 96 \\
Nueva SS & $2,533,232$ & 20,739 & 122 \\
San Vicente & $1,719,064$ & 139,204 & 12 \\
Zacatecoluca & $1,410,119$ & 13,783 & 102 \\
San Miguel & $4,427,323$ & 27,126 & 163 \\
Usulután & $1,262,294$ & 11,560 & 109 \\
Zacamil & $3,118,289$ & 15,358 & 203 \\
\hline
\end{tabular}

Fuente: elaborado con base en la información del Diario Oficial del 22 de diciembre de 1995.

\section{Costos estándar}

Si se asumieran los costos encontrados para el caso mexicano, se podría establecer que los costos institucionales se elevarían notablemente, pasando hasta los $155 \mathrm{mi}-$ llones de dólares( 1,197 dólares por 78,726 lesionados). Al considerar el costo propuesto para sociedades más desarrolladas, como la de Estados Unidos 5 , los costos se elevan aún más pudiendo llegar inclusive a multiplicarse por diez.

\section{Costos de rehabilitación}

Otro costo directo de la violencia está íntimamente relacionado con la atención de los sobrevivientes de la violencia que requieren de terapias posteriores a su egreso del hospital, es decir, que causan costos institucionales no solamente en el área de hospitalización, sino también en lo referente a la rehabilitación. En El Salvador, el Instituto Salvadoreño de Rehabilitación de Inválidos (ISRI) desarrolla las funciones de rehabilitación, tanto de los lisiados por la violencia como de pacientes que sufren de complicaciones congénitas. Como muestra, el Cuadro 6 muestra que la mayor parte del presupuesto del ISRI es dedicado a las áreas de servicios de salud y, dentro de estos, los nubros más relacionados con la violencia son los que se refieren a la "atención de aparato locomotor" y la "rehabilitación profesional", con un costo promedio de 136.1 y 1,360.1 dólares. En total, ambos rubros implicaron costos directos por un monto de 639,572 dólares.

5. Para el caso de la ciudad de México se han establecido los siguientes valores promedio para 1995: costo promedio por individuo, 1,971 dólares; lesiones leves, desde 28 a 400 d6lares; lesiones graves, desde 400 a 8,000 dólares; complicaciones, más de 25,000 dólares (Fundación Mexicana para la Salud y Centro de Economía y Salud, 1997. pp. 28 y 29). 


\section{Cuadro 5}

Instituto Salvadoreño de Rehabilitación de inválidos y destino del gasto

\begin{tabular}{lcc}
\hline Unidad presupuestaria & Monto asignado & Porcentajes \\
\hline Dirección y administración & $546,998.3$ & 14.9 \\
Servicios de salud & $3,124,999$ & 85.1 \\
\hline Total & $3,671,997$ & 100.0 \\
\hline
\end{tabular}

Fuente: elaborado con base en los datos del Diario Oficial de 22 de diciembre de 1995, p. 487.

\section{Cuadro 6}

Unidad presupuestaria de los servicios de salud y relación propósitosrecursos en rehabilitación integral*

(En dólares)

\begin{tabular}{lrrr} 
Propósito & Meta & Costo & Costo unitario \\
\hline Atención de ancianos & 230 & 635,246 & $2,761.94$ \\
Atención a discapacitados visuales & 220 & 272,218 & $1,237.35$ \\
Educación especial niños Down & 450 & 248,368 & 551.93 \\
Atención pacientes aparato locomotor & 3,500 & 476,364 & 136.10 \\
Tratamiento de audición y lenguaje & 1,500 & 276,805 & 184.54 \\
Atención de parálisis cerebral & 800 & 185,635 & 232.04 \\
Atención invalideces múltiples & 2,460 & 215,735 & 87.7 \\
Rehabilitación integral, región occidental & 4,650 & 241,566 & 51.95 \\
Rehabilitación integral, región oriental & 3,800 & 249,011 & 65.53 \\
Rehabilitación profesional & 120 & $163,208.6$ & $1,360.08$ \\
Consulta externa & 1,000 & 84,568 & 84.57 \\
Descentralización de servicios & 500 & 76,273 & 152.55 \\
\hline Total & & $3,125,000$ &
\end{tabular}

* Instituto Salvadoreño de Rehabilitación de Inválidos.

Fuente: elaborado con base en los datos del Diario Oficial del 22 de diciembre de 1995.

\subsection{Sector seguridad}

\section{A. Costos asociados con la prevención}

Como resultado de la proliferación de la violencia, en los últimos años han proliferado diferentes actividades emprendidas tanto por el sector público como el privado, tendientes al control y prevención de la violencia. Por un lado, el gobierno ha respondido con la creación de una unidad de política criminal en el interior del Ministerio de Justicia la cual teóricamente es la encargada de diseñar e implementar planes de prevención y mitigación de la violencia. Por otra parte, durante los últimos años han proliferado las compañías de seguridad privada, las cuales ofrecen los servicios de prevención y, en algunos casos, atención de las secuelas de la violencia. Un estimado de los costos en que incurre la sociedad por pago de seguridad privada surge de considerar el costo horario promedio del servicio (2.86 dólares), multiplicarlo por las 24 horas del día y, luego, por el total de agentes disponibles en un día promedio que se estima está rondando los 7,000 agentes, lo cual equivale a 7,000 turnos para las 24 horas del día. En total, los gastos de seguridad privada para control y prevención de la violencia estaría cercano a los 7,207,202 dólares. 


\section{B. Costos legales}

Los costos legales están relacionados con los gastos ejecutados por el gobierno en materia judi- cial, policial y carcelaria, estos se presentan en el Cuadro 7. Como podrá notarse, cuantitativamente, estos son los mayores costos directos de la violencia.

Cuadro 7

Presupuesto policial-judicial-carcelario

\begin{tabular}{lrr}
\hline Rubro & Presupuesto & Porcentajes \\
\hline Organo Judicial & $87,223,379$ & 31.0 \\
Fiscalía General de la República & $6,729,309$ & 2.4 \\
Procuraduría para la Defensa de los Derechos Humanos & $3,427,013$ & 1.2 \\
Ramo de Seguridad Pública & $154,231,610$ & 54.9 \\
Ramo de Justicia* & $13,360,456$ & 4.8 \\
Academia Nacional de Seguridad Pública & $15,982,021$ & 5.7 \\
\hline Total & $280,953,780$ & 100 \\
\hline
\end{tabular}

* Incluye la Dirección General de Centros Penales y de Readaptación.

Fuente: elaborado con base en los datos del Diario Oficial del 22 de diciembre de 1995, pp. 117, 131, 148, 174 y 185.

Sólo para el mantenimiento de la actividad policial (Ramo de Seguridad Pública y Academia Nacional de la Seguridad Pública) se canalizó una suma superior a los 170 millones de dólares para 1996, representando cerca de 60.6 por ciento del total de los costos legales. El mantenimiento de la actividad judicial absorbe un monto cercano a los 107 millones de dólares equivalentes a un 38.2 por ciento del total de los costos legales. En el presupuesto de la actividad judicial se incluye el sistema carcelario, el cual absorbe un total 9,711,670 dólares. El restante 1.2 por ciento de costos legales se dedica a la atención de las implicaciones judiciales y legales de casos de violencia. En total, los costos legales implican un costo de $280,953,780$ dólares equivalente a un 4.9 por ciento del Producto Interno Bruto, lo cual resulta claramente superior al total de poco más de 47 millones erogados en concepto de costos directos para la recuperación de la salud.

\section{Costos particulares}

De acuerdo con los resultados de la encuesta de investigación, de los costos de la violencia rea- lizada para esta investigación, además de los gastos en servicios de seguridad, los costos de la violencia también comprenden los gastos en que incurren los hogares para instalar mecanismos de seguridad y para contratar servicios de vigilancia locales o "serenazgos".

Según la encuesta, los costos de los mecanismos de seguridad se encuentran rondando el promedio de 54.8 dólares por hogar. En total, el AMSS comprende un total de 323,400 hogares lo cual implica un gasto total de $17,722,320$ dólares. Para el resto de hogares del país no existen datos que permitan calcular el promedio de gastos en mecanismos de seguridad y los costos totales en este concepto.

Los gastos en servicios de serenazgo se estiman en un promedio de 25.56 dólares anuales, lo cual implica un gasto total de 8,266,104 dólares para el AMSS. Para el resto de hogares del país $(846,054)$ se estima un gasto anual de 4.32 dólares $^{6}$ lo cual implica un gasto de 3,654,953 dólares. En total, los gastos de serenazgo implicarían un total de 11,921,057 dólares.

6. Calculado con base en la Encuesta Nacional de Ingresos y Gastos (Ministerio de Planificación y Coordinación del Desarrollo Económico y Social, 1992). 
En conjunto, los gastos en mecanismos de seguridad y de serenazgo efectuados por los hogares salvadoreños en 1995 habría sido de 29,643,377 dólares.

\section{Costos indirectos}

\subsection{Producción e ingreso (ingreso perdido por grupo ocupacional)}

Al considerar los datos ofrecidos por las Encuestas de Hogares de Propósitos Múltiples es posible obtener algunos indicadores de los ingresos futuros que se pierden por efecto del fallecimiento del agente productivo o por efecto de la disminución de sus capacidades físico-mentales. Por ello, para estimar el ingreso perdido por efecto de la violencia se ha analizado cual es el efecto sobre el ingreso que pudo haber sido generado ya sea por una persona fallecida o por una persona que ha sufrido disminución de sus capacidades.

Los primeros datos de Años de Vida Saludables Perdidos (AVISA) por efecto de muertes violentas y años vividos con discapacidad se estima en 182,631 años. Considerando que el promedio de ingresos y salarios de los hombres, que concentra la mayoría de los homicidios violentos, es de 2,714.4 dólares anuales tendríamos que, en total, los ingresos perdidos por fallecimientos violentos sería de 495,733,583 dólares. Al actualizar esta ci- fra mediante el método del valor actual se tiene que los ingresos perdidos por efecto de los fallecimientos para 1995 se encuentran cercanos a los $169,501,778$ dólares.

\subsection{Pérdidas de la economía}

Como resultado de la violencia, el Estado se ve obligado a dedicar buena parte de su presupuesto para cubrir los gastos asociados a la atención de las víctimas de la violencia, pero más aún para cubrir los costos legales asociados con ella. Si consideramos en su conjunto los costos de la violencia tenemos que en conjunto los costos legales e institucionales alcanzan los 284,304,710 dólares, equivalentes a un 16.8 por ciento del tolal del presupuesto de la nación y a un 67.5 por ciento de la inversión pública.

La inversión privada también se ha visto fuertemente limitada por la violencia, prueba de ello es la situación suscitada en El Salvador a principios de la década pasada cuando, a causa del recrudecimiento de la guerra civil, hubo fugas masivas de capital y caída de la inversión. Evaluar el efecto de la violencia en 1996 resulta más complicado en tanto que no se cuenta con las series de datos para aplicar técnicas de regresión (magnitud de la violencia, costos directos e indirectos, etc.) que permitan estimar los efectos de la violencia sobre la inversión privada; sin embargo, puede recurrirse a ejercicios contables para establecer un estimado.

Cuadro 8

Pérdidas materiales asociadas a la violencia (En dólares)

\begin{tabular}{lcr} 
Tipo de violencia & Pérdida económica & Costos asociados \\
\hline Hurto y robo & $70,826,241$ & $5,830,314$ \\
Hurto y robo de vehículos & $1,990,894.5$ & 393,813 \\
Delitos contra el patrimonio & $150,552,400$ & $627,827.2$ \\
Total por rubro & $223,369,535$ & $6,851,954$ \\
\hline
\end{tabular}

Total general

$230,221,489$

Fuente: elaboración propia. 
Una forma podría ser considerar los costos que en materia de costos directos personales y costos de prevención son erogados para obtener una idea de los recursos de ahorro que se divierten para sufragar parcialmente los costos de la violencia. En total, los dos costos mencionados totalizan los 11,931,325 dólares, sin embargo, no debe soslayarse el hecho de que la mayor parte de los costos de prevención representa un cargo para los costos empresariales $\mathrm{y}$, en esa medida, un recargo sobre la inversión privada. En cambio los costos directos de atención de la salud son absorbidos principalmente por grupos familiares que sacrifican consumo.

Si se consideran solamente los costos de la prevención, tendríamos que la inversión privada se habría visto reducida en sólo un 0.5 por ciento por efecto de los costos erogados en la prevención. Por otra parte, los gastos de consumo apenas se habrían reducido en un 0.05 por ciento del Producto Interno Bruto. De haberse canalizado estos recursos a la inversión pública, el nivel esperado para 1996, el PIB habría sido superior en un 0.2 por ciento al efectivamente logrado para esos años.

\subsection{Pérdidas materiales}

Por efecto de la violencia también se reportan importantes costos indirectos relacionados con la pérdida de bienes o valores. De esta forma, la en- cuesta realizada para conocer las pérdidas como producto de la delincuencia señala un promedio de 205.3 dólares como pérdida por el robo. Por otra parte, ateniéndose a lo señalado en el artículo "Magnitud de la violencia en El Salvador" (en este mismo número), se tendría que un 29.5 por ciento de los hogares habría sido víctima de robo o hurto, lo cual implicaría, para el total del país, un total de 344,989 hogares. De los datos anteriores se obtiene que las pérdidas por robo y hurto alcanzarían los 70,826,241 dólares. Adicionalmente se incurriría en un costo promedio de 16.9 dólares por efecto de los robos o hurtos, lo que implica un gasto adicional de 5,830,314 dólares.

Para el caso de robo y hurto de vehículos, la encuesta encontró que el promedio del valor de vehículos y accesorios de vehículos hurtados está cercano a los 902.9 dólares mientras que los gastos asociados a los mismos promediarían los 178.6 dólares. De acuerdo con datos de la Fiscalía General de la República, el robo de vehículos estaría rondando cerca de 2,205 casos para el año 1995, con lo cual se obtiene que las pérdidas monetarias por hurto y robo de vehículos fue de $1,990,894.7$ dólares, mientras que los gastos asociados a este tipo de delito fueron de 393,813 dólares.

Los delitos contra el patrimonio acusan niveles de pérdidas mucho mayores que los dos casos an-

\section{Cuadro 9}

\section{Costos totales estimados como producto de la violencia}

\begin{tabular}{|c|c|c|c|}
\hline Rubro & Cifras en dólares & Porcentajes & $\begin{array}{c}\text { Porcentajes del PIB } \\
(5,774,000,000.6 \text { dólares })\end{array}$ \\
\hline \multicolumn{4}{|l|}{ Costos directos } \\
\hline Costos directos personales & $56,646,377$ & 7.28 & 0.98 \\
\hline Costos directos intitucionales & $20,382,161$ & 2.62 & 0.35 \\
\hline Costos de rehabilitación & 639,572 & 0.08 & 0.01 \\
\hline Costos asociados con la prevención & $7,207,202 *$ & 0.93 & 0.12 \\
\hline Costos legales & $280,953,780$ & 36.14 & 4.87 \\
\hline \multicolumn{4}{|l|}{ Costos indirectos } \\
\hline Ingresos perdidos & $169,501,778$ & 21.80 & 2.93 \\
\hline Pérdidas a la economía & $11,931,325$ & 1.53 & 0.21 \\
\hline Pérdidas materiales & $230,221,489$ & 29.61 & 3.99 \\
\hline Total & $777,483,684$ & 100 & 13.46 \\
\hline
\end{tabular}

* Anuales, suponiendo tarifa de 2.86 dólares por hora y la disponibilidad de 7,000 tumos para las 24 horas del día. 
teriores, y se observa un promedio de pérdidas de 10,743 dólares. Sin embargo, los costos asociados son relativamente bajos con un promedio de sólo 44.8 dólares. Las proyecciones de la encuesta permiten estimar un aproximado de 14,014 casos de delitos contra el patrimonio en 1995 lo cual implicaría costos por el orden de $150,552,400$ dólares por efecto de las pérdidas y de $627,827.2$ dólares por costos asociados.

\section{Costos totales}

El Producto Interno Bruto de El Salvador para 1995 ascendió a 5,774,000,000.6 dólares estadounidenses. Los datos presentados y resumidos en el Cuadro 9 permiten establecer que en 1995 se gastó un aproximado de setecientos setenta y siete millones cuatrocientos ochenta y tres mil seiscientos ochenta y cuatro dólares estadounidenses (777,483,684 dólares) como producto de la violencia en El Salvador. Eso significa que más del 13 por ciento del Producto Interno Bruto en 1995 se utilizó para cubrir los diferentes costos de la violencia. Considerando que el crecimiento económico para ese año fue del 6.3 por ciento, es posible afirmar que, por efecto de la violencia, se pierde una proporción del PIB que es dos veces mayor lo que éste se incrementa por efecto del aumento de la inversión, el consumo y/o las exportaciones.

\section{Referencias}

Banco Central de Reserva. Revista trimestral, enero-marzo, San Salvador, El Salvador, 1996.
Bobadilla, J.L.; Cárdenas, V.; Couttolenc, B. et al. Medición de los costos de la violencia. Resultados de un taller organizado por la Organización Panamericana de la Salud (OPS) y el Banco Interamericano de Desarrollo (BID) Caracas, 11-13 de diciembre, 1995.

Dirección de Información. "Resultados de la Encuesta de Hogares de Propósitos Múltiples", San Salvador, El Salvador, Documento mimeografiado, 1997.

Dirección de Información. Encuesta de Hogares de Propósitos Múltiples, San Salvador, El Salvador, Ministerio de Relaciones Exteriores, 1996.

Dirección de Información. Encuesta de Hogares de Propósitos Múltiples, San Salvador, El Salvador, Ministerio de Relaciones Exteriores, 1995.

Fundación Mexicana para la Salud y Centro de Economía y Salud. "La violencia en la ciudad de México: análisis de la magnitud y su repercusión económica (Informe preliminar)", Ciudad de México, Documento mimeografiado, 1997.

Ministerio de Hacienda. Ley de Presupuesto General del Estado para el año 1996 por áreas de gestión, San Salvador, El Salvador, 1996.

Ministerio de Salud Pública y Asistencia Social. "Boletín de prensa sobre los costos que implica para el Ministerio de Salud, atender a los pacientes que demandan servicios de emergencia producto de la violencia", San Salvador, El Salvador, Documento mimeografiado, 1997. 